\title{
COMPETENCIAS DE INVESTIGACIÓN EN ESTUDIANTES Y ESTUDIOS UNIVERSITARIOS DE TRABAJO SOCIAL EN ESPAÑA
}

\section{Research competences in University students and studies of Social Work in Spain}

\author{
José JAVIER NAVARRo PÉREZ ${ }^{1}$ \\ MARÍA DE LAS MERCEDES BOTIJA YAGÜE ${ }^{2}$
}

\begin{abstract}
Resumen
Este documento analiza la adquisición de competencias en investigación en los estudios de Grado de Trabajo Social de la Universidad de Valencia (España). Los cambios del nuevo orden social exigen una actualización continua tanto de los métodos como de las técnicas que guían la acción de los titulados universitarios. Esta investigación compara las competencias de investigación / intervención entre alumnos de cuarto y último curso de Grado en Trabajo Social con profesionales diplomados en Trabajo Social reciclados para realizar el curso de actualización al Grado. La fiabilidad de los resultados obtenidos se corrobora mediante un método test-retest con la realización de un taller práctico que mejoró las competencias de los estudiantes ante el diseño de proyectos de investigación social. Las conclusiones del artículo informan de la importancia de los proyectos de investigación social (PIS) para los profesionales del Trabajo Social y la necesidad de generar diagnósticos altamente fiables para intervenir con eficacia.
\end{abstract}

Palabras clave: Proyectos de Investigación Social; diagnóstico social; competencias; nuevo Trabajo Social; Perfiles Educativos

\section{Extended Abstract}

This document analyses the acquisition of research skills on the Degree in Social Work of the University of Valencia (Spain). Changes in the new social order mean that the methods and techniques that guide the actions of university graduates have to be constantly updated. The present investigation compares

\begin{abstract}
This document analyses the acquisition of research skills on the Degree in Social Work of the University of Valencia (Spain). Changes in the new social order mean that the methods and techniques that guide the actions of university graduates have to be constantly updated. Social changes allow new methods and techniques applied for college students. The present investigation compares the research and intervention skills of students on the fourth and final year of the Degree in Social Work with those of social work graduate professionals undergoing retraining on, for the purposes of planning the degree update course. The reliability of the results validated using the test-retest method, whereby a practical workshop was held in which the improvements in the students' skills in designing social research projects could be observed. The conclusions highlight the importance of social research projects for social work professionals and the need to generate highly reliable diagnoses for intervention to be effective.
\end{abstract}

Keywords: Social research projects; competences; social diagnosis; new Social Work; Educational Profiles.

the research and intervention skills of students on the fourth and final year of the Degree in Social Work with those of social work graduate professionals undergoing retraining on, for the purposes of planning the degree update course. The reliability of the results validated using the test-retest method, whereby a practical workshop was held in which the improvements in the students' skills in designing

1. Profesor Contratado Doctor. Departamento de Trabajo Social y Servicios Sociales. Facultad de Ciencias Sociales. Universidad de Valencia. Campus Tarongers s/n ${ }^{\circ}, 46021$, Valencia. Despacho 2 D23, Telf. 963828193 / 9638282 04. Correo electrónico: J.Javier.Navarro@uv.es

2. Profesora Ayudante Doctora. Departamento de Trabajo Social y Servicios Sociales. Universidad de Valencia. 
social research projects could be observed. The conclusions highlight the importance of social research projects for social work professionals and the need to generate highly reliable diagnoses for intervention to be effective.

\section{Design and procedure}

The work included different sub procedures. First of all an extensive bibliographical search was carried out regarding the design of social research projects and research funding competitions at local, national and international level. The curricula of different universities were then analysed, focusing on the training and skills taught for the design of research projects. Finally a sample of 78 subjects was obtained. This was split into two groups of 39 individuals, one comprising professionals with social work experience and the other comprising final-year students on the Degree in Social Work (with no professional experience).

Once the sample was organized, participants were given an ad hoc questionnaire to measure skills and assess the presence/absence of other skills relating to diagnosis and intervention plans. A feedback questionnaire was administered at the end and a retest carried out enabling the research and intervention skills acquired to be established.

\section{Results}

The main contributions of the work concern the abilities in research skills of new social work students, both those entering the field for the first time and those on refresher courses. A high percentage of both groups believe that, in order to intervene, a prior analysis of the situation needs to be carried out, a diagnosis made and successful interventions planned. In this respect the professionals are perceived as focusing their efforts on intervention, the reason for this being the lack of time they have to dedicate to research and diagnosis in practical day-today cases. The bureaucratization of social work tasks makes it difficult to draw up diagnostic criteria that include comparative assessments.

As far as assessing research skills is concerned, both groups thought that those involving intervention were the most important, followed by those relating to methodology and scientific transfer or productivity deriving from professional practice. As for their perception of acquired skills, the professionals felt less sure about methodological research skills while the degree students were less confident with skills involving intervention.

Both groups were interested in experimental methods in social work. Comparing the skills of both groups, connections were found in the development of skills to analyse and systematize data. The main differences concerned the priority given to intervention to the detriment of research, even though the latter was perceived as crucial for producing successful intervention plans. While the final-year students were less sure about intervention and more confident about research, the results for the professional students were the opposite. The refresher students were also better able than the degree students to relate social work theory to pre-diagnostic professional intervention. As for skills deriving from immersion in bibliographical databases, the degree students gained higher scores and tackled these exercises with greater diligence and practice than the professionals. The professionals stressed the need to retrain in order to acquire new research skills.

A subsequent retest that was carried out to assess the skills acquired by both groups showed that around $80 \%$ of both degree students and professionals correctly set out intervention plans, although only $60 \%$ of the professional collective and $40 \%$ of the student collective reached an acceptable level in research planning. The professionals underestimated their own skills whereas the final-year students overestimated their own knowledge.

\section{Conclusions}

The main conclusions drawn from this investigation are that:

1. Training in research is a priority. It is important to design a Degree in Social Work in which research subjects take on greater relevance in the future.

2. Research is a stage that precedes and is inseparable from the intervention process.

3. Systematizing the practical aspect makes it possible to reflect on professional activity.

4. The professionals perceive research methodology to be more difficult and are more confident when dealing with social contingencies and new social vulnerabilities. The non-professional students are more secure when acquiring research skills, but have less success than the professionals when tackling empirical work. The degree students' expectations of learning are therefore higher than the professionals', although the evidence does not match their perceptions. The professionals showed correspondingly less insecurity in intervention and research than the final-year degree students.

Professional skill in researching and dealing with the social reality is one of the main results of this investigation - better research skills are needed because this will make it easier to analyse the social reality and make it possible to set up intervention mechanisms tailored to social and personal needs. This will enable the challenge of exploring the relationship between theory and practice to be recovered, along with the strategies of research - action - transformation. 


\section{Introducción}

El presente trabajo tiene por objeto evaluar la adquisición de competencias de investigación e intervención de los estudios universitarios de Trabajo Social, adaptando este análisis al alumnado de la Universidad de Valencia.

Los nuevos estudios de Grado en Trabajo Social tienen ante sí un importante abanico de posibilidades en referencia a la investigación. El Trabajo Social, se determina como perfil educativo que mantiene una importante tradición en la formación práctica para la intervención (Moix, 2006). A partir de la gestación de los nuevos planes de estudio, se afianzan los contenidos en investigación. Mediante el reconocimiento del grado, nos situamos con capacidad para definir y orientar nuestro marco conceptual y las líneas investigadoras propias de lo que venimos a definir como el nuevo Trabajo Social. Como decimos, los estudios de Grado en Trabajo Social inciden en la elaboración y defensa del Trabajo Final de Grado (en adelante TFG) que se presenta obligatorio en once de los veintiséis países de la UE: Alemania, Dinamarca, Eslovenia, Estonia, Francia, Grecia, Holanda, Italia, Lituania, Noruega y Finlandia. A pesar de la divergencia de modelos de bienestar que caracterizan estos países, William y Nelson (2007) inciden en la convergencia del tipo de trabajo que debe culminar el estudiante de Trabajo Social para ser competente el ejercicio de la profesión. En este sentido, el TFG tiene como objetivo la integración teórico-práctica y la iniciación a la investigación sobre la realidad profesional del Trabajo Social.

Durante mucho tiempo, la enseñanza del Trabajo Social en España se centró en formar diplomados universitarios capaces de gestionar el espacio profesional, y en consecuencia, según Ahmed (2009: 28), “manejándose con marcos teóricos de análisis y de intervención que fueron propuestos para realidades muy distintas a nuestra contemporaneidad". Los actuales estudios universitarios de Trabajo Social, han crecido como disciplina no solo en la gestión de recursos y en la construcción de identidad y ética propia, sino que además han evidenciado una gran transformación desde el punto de vista empírico alentada por las tesis doctorales leídas, con mayor incidencia si cabe en los últimos años (Pérez-Cosin, 2003 y Miranda, 2003; Pastor, 2008; Martín, 2013; Fernández, 2015 y Álvarez, 2015).

La realidad que rodea la práctica del Trabajo Social se ha visto modificada por las problemáticas globales, "obligando a diversificar la práctica" (Barner y Okech, 2013: 1062) y actualizando técnicas y procedimientos. En este sentido, se pone de manifiesto la necesidad de reformular los procesos de resolución de problemas sociales y las dificultades que afectan a las personas. A este respecto Brydon (2012) apunta a la complejidad de la práctica del Trabajo Social, pues 
los nuevos conflictos exigen nuevas respuestas y sobre ellas han de construirse los procedimientos para descubrirlas.

\section{El Espacio Europeo de Educación Superior (EEES): oportunidad para el Trabajo Social}

El EEES ha generado un proceso de cambio en distintas universidades europeas. La firma de la Declaración de Bolonia con la presencia de 29 Gobiernos Europeos, sirvió para asentar los cimientos y posibilitar la edificación de un espacio común en lo que se refiere a estudios universitarios. Para las universidades españolas, esto supuso la incorporación a la excelencia de las titulaciones, generando la aceptación de un nuevo modelo de enseñanza-aprendizaje centrado como presenta el libro Blanco del Trabajo Social en la adquisición de competencias por parte del alumnado y el establecimiento de un sistema internacional de créditos.

El Sistema Europeo de Transferencia de Créditos (en adelante ECTS) cuantifica no sólo las horas de clase teóricas sino también el trabajo no presencial que debe realizar el alumnado. De esta manera, los estudiantes tienen la posibilidad de participar en el diseño de su aprendizaje. Compartimos con Medina, Domínguez y Sánchez (2013) que el aprendizaje basado en competencias implica evolucionar desde un planteamiento centrado en el profesor y la exposición de clases magistrales a otro orientado al estudiante y a sus potencialidades para implementar autónomamente los contenidos en la práctica real. Así pues "la formación por competencias reorienta las decisiones didácticas en relación con la enseñanza, aprendizaje y evaluación, centrándolo en el estudiante, sus procesos y resultados de aprendizaje" (García-San Pedro, 2009:11). Esto supone que la investigación en Trabajo Social deberá desarrollarse en relación con la práctica e "incorporarse en el marco académico que legitima la disciplina" (Campanini, 2009: 113).

Los ECTS implican el establecimiento de estrategias sobre las que vincular la enseñanza teórica con la práctica, enlazando el mundo académico con el posterior medio laboral en que ha integrarse el alumnado. Este concepto de universidad que gira a lo largo del ciclo vital, permite la interacción con el espacio profesional, posibilitando nuevas vías de acceso a la investigación que hasta hace pocas décadas quedaban prácticamente reservadas al espacio académico. En esta línea Liedgren (2015) apunta la importancia del docente para investigar la práctica desde dentro de la misma.

En consecuencia, la realización de esta tarea exige a los estudiantes un elevado nivel de aptitudes que le permitan desempeñar su práctica profesional para la que además se han formado. Nos situamos pues en un nuevo 
escenario donde el concepto competencia adquiere un relevante valor y que Zabalza (2002: 12) definió como "elemento en el que se pone acento en los resultados del aprendizaje, en lo que el alumno es capaz de hacer al término del proceso educativo y en los procedimientos que le permiten continuar aprendiendo de forma autónoma a lo largo de la vida”. Por ello es interesante integrar en un único cuerpo tres conceptos armónicos que han de correlacionar entre sí: el saber ser (atendiendo a las aptitudes intrínsecas al individuo), el saber conocer (en la forma de capacidades para observar, expresar, comprender y analizar) y el saber hacer (que incluirá las posibilidades para el desempeño basado en procedimientos y potencialidades para proyectar las habilidades adquiridas); estos tres saberes, deberán simultáneamente advertir las contingencias del entorno, las necesidades personales y los procesos de incertidumbre, con soberanía intelectual, autonomía para la crítica, carácter creativo... etc. En relación a ello, convenimos con Vázquez (2011), al plantear que la conjunción de estos tres saberes, habrá de garantizar la capacitación de los estudiantes para proyectar las competencias para las que han sido entrenados. Estas son resultado de un proceso que requiere de la transversalidad y complementariedad de las distintas guías docentes que componen el título de Grado en Trabajo Social.

Coincidimos con Díaz Barriga (2014) que las competencias deberán integrar resultados relativos a la adquisición de conocimiento, valores y habilidades necesarias para la práctica. De acuerdo con esto, Chourides, Longbottom y Murphy (2003) la gestión del conocimiento hacia la excelencia se ha convertido en un fenómeno con altas implicaciones para la innovación y la competitividad en los últimos tiempos. Es lo que venimos a definir como el aprendizaje permanente: más cercano a la trasferencia y a la interacción del alumnado con la sociedad. La Universidad no es lugar para unos pocos privilegiados, sino que tal como refieren Biggs y Tang (2013), el conocimiento se encuentra más allá del espacio que encierran las aulas.

En esta línea, y tal como dispone el Libro Blanco del Trabajo Social (2005), entre los principales argumentos de desempeño que han de adquirir los estudiantes en formación serán la capacidad para demostrar competencia profesional en el ejercicio del Trabajo Social, incentivando para ello tal como describe la competencia 22 "Investigar, analizar, evaluar y utilizar el conocimiento actual de las mejores prácticas de trabajo social para revisar y actualizar los propios conocimientos sobre los marcos de trabajo" (2005: 191). En correlación directa con estos elementos, uno de los mejores instrumentos en el que podemos observar esta adquisición de competencias se produce al diseñar el proyecto de investigación social (en adelante PIS). Desde esta perspectiva, se concibe el PIS, como una oportunidad de conectar el objeto de investigación 
con el diagnóstico, y en consecuencia con una intervención posterior que invierta en dinámicas de transformación tanto propias del sujeto como colectivas (Pastor y Sánchez-Millán, 2014).

Figura 1: Configuración del PIS

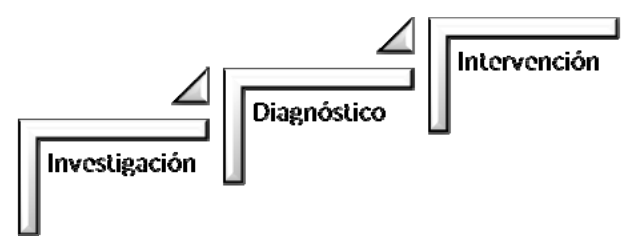

Fuente: Elaboración propia.

Es decir, el PIS permite presentar un protocolo diversificado de actuaciones que permitan diseñar intervenciones diagnósticas. Por tanto, permite proporcionar el proceso de intervención a la realidad social.

\section{Metodología}

En el plano metodológico para abordar el fenómeno presentado, nos situamos en la pluralidad de técnicas y perspectivas de análisis para analizar la realidad desde diferentes puntos de vista (Bourdieu, 1999). En este sentido se realizó un estudio sobre dos grupos: por un lado los profesionales, trabajadores/as sociales en activo que se encontraban realizando el curso de adaptación o nivelación al Grado y por otro, aquellos estudiantes del nuevo Grado en Trabajo Social matriculados en cuarto y último curso de la titulación.

Tal como plantean Álvarez, et al. (2001: 616) "la universidad es un excelente campo abierto a la investigación" y en consecuencia, nos planteamos valorar las capacidades de los distintos perfiles de estudiantes una vez realizado el taller de PIS para posteriormente establecer un análisis comparativo entre ambos grupos. El objetivo general tal como enunciamos al principio del documento, trató de examinar la adquisición de competencias en investigación e intervención de los matriculados.

En consonancia con los mecanismos de ajuste utilizamos una metodología multimétodo que integró los siguientes procesos:

- En primer lugar se realizó una importante búsqueda bibliográfica relativa a diseño de proyectos de investigación social, así como convocatorias de ayudas para la investigación a nivel local, nacional e internacional. 
- En segundo lugar se analizaron los planes de estudios de diferentes universidades en lo referido a la carga lectiva (número de créditos ECTS), dedicada al diseño de proyectos de investigación.

- En tercer lugar, sobre una población total de 183 estudiantes se obtuvo una muestra de 78 sujetos, que se dividió en dos grupos de 39 individuos; por un lado los profesionales de nivelación o adaptación al Grado $^{3}$ (con experiencia como trabajadores sociales) y por otro los estudiantes de último curso de Grado en Trabajo Social (obviamente sin experiencia profesional).

Para la selección muestral se siguieron los siguientes criterios de inclusión:

- Para el grupo de nivelación: haber superado la asignatura de Investigación Diagnóstica y desarrollado en el ejercicio profesional al menos 800 horas. Se optó por esta selección muestral dado que el cuestionario preguntaba sobre las competencias en investigación que habían sido adquiridas durante el curso. Relativo al mínimo de horas exigido, se tomó como referencia el acuerdo de la Comisión Académica de Título (CAT) para la convalidación por este perfil específico de alumnado, de las asignaturas Técnicas y Procedimientos y Proyectos Sociales.

- En relación con el alumnado de Grado, se especificó como partícipes de la muestra haber superado las asignaturas troncales relativas a la investigación e intervención en Trabajo Social y estar matriculado en el Trabajo Final de Grado (TFG), atendiendo que la actividad de Taller se desarrolló para el abordaje del mismo.

A ambos grupos se les pasó el mismo cuestionario pre test con preguntas tanto cerradas como abiertas. En este se analizaba el interés por la investigación así como su relación con el diagnóstico y la intervención. Posteriormente se valoraba la importancia de la adquisición de competencias en materia de investigación y finalmente, mediante unas preguntas de control se trató de verificar la adquisición de diferentes competencias en el diseño de proyectos de intervención y de investigación. Con ello se obtuvo un marco referencial de necesidades en materia de investigación / intervención. En esta línea, Rodríguez (2000) recomienda la implementación de talleres colaborativos -en este caso,

3. Profesionales que habían cursado previamente la titulación acogidos a planes de estudios anteriores en la forma de Diplomatura en Trabajo Social y que deseaban nivelar (adaptar) su formación al nuevo plan de ordenación académica de los títulos universitarios españoles (Grados Universitarios). 
orientado al diseño de PIS- pues favorecen los resultados de aprendizaje curricular. Posteriormente se pasó un cuestionario de satisfacción y se realizó un re-test mediante el que se pudo comprobar las competencias adquiridas en investigación e intervención y el efecto que supuso el en el alumnado la asistencia y participación en el Taller para el diseño del PIS y el abordaje del TFG.

Centramos nuestra atención principalmente en aquellas variables relativas al interés que la investigación pre diagnóstica despertó en los estudiantes. Posteriormente nos adentramos en aquellas cuestiones destinadas a informar sobre el proceso para la adquisición de competencias, finalizando con un conjunto de preguntas evaluativas con el objetivo de identificar el grado de comprensión e interiorización de contenidos curriculares para el diseño, investigación e implementación de proyectos de intervención social y educativa. Los resultados de ambos grupos por separado fueron interesantes para realizar un análisis comparativo y fruto de este trabajo, planteamos elaborar un artículo científico.

Esta experiencia se alinea con los trabajos de McKeachie (2002) relativos al aprendizaje colectivo por procesos de investigación acción, pues nos permite poner en valor una serie de actividades y talleres construidos sobre metodologías de aprendizaje colaborativo. El uso de estos enfoques alentó el dinamismo, el trabajo en equipo y la gestión colectiva de las problemáticas desde la unidad de grupo aula.

Significar finalmente que la utilización de los resultados se planteó con consentimiento ético de los participantes. Además, garantizamos la confidencialidad de toda la información y opiniones que los sujetos presentaron a través del cuestionario, basado en el código de ética de los titulados en Trabajo Social y de los trabajadores sociales (Texto aprobado por la Asamblea General de las asociaciones de licenciados en trabajo social y asistentes sociales de España, en la sesión extraordinaria que se celebró el 12 de junio de 2012). Y bajo la ley Orgánica 15/1999, de 13 de marzo por la Ley de protección de datos personales (LOPD).

\section{Resultados: La relevancia de la investigación}

A medida que se amplía nuestra mirada a los actuales estudios universitarios de Grado en Trabajo Social, los resultados derivados del análisis inciden en que tanto el PIS como el diagnóstico son considerados de gran relevancia en la articulación de los planes de estudio de las titulaciones de Trabajo Social en España. En todos los Grados universitarios se favorece la adquisición de competencias en esta materia y en un 70\%, el PIS cumple la función de Trabajo Fin de Grado. 
En referencia a los hallazgos relativos a bibliografía de proyectos sociales y convocatorias de ayudas, cabe destacar que la información relativa al PIS es menos abundante que la relacionada con planificación, gestión e intervención. Relativo a convocatorias públicas y/o competitivas para la financiación de proyectos de investigación, hallamos algunas diferencias en el diseño de proyectos de investigación social, no obstante pudiéndose abstraer un marco genérico básico ${ }^{4}$.

Figura 2: Estructura básica del PIS

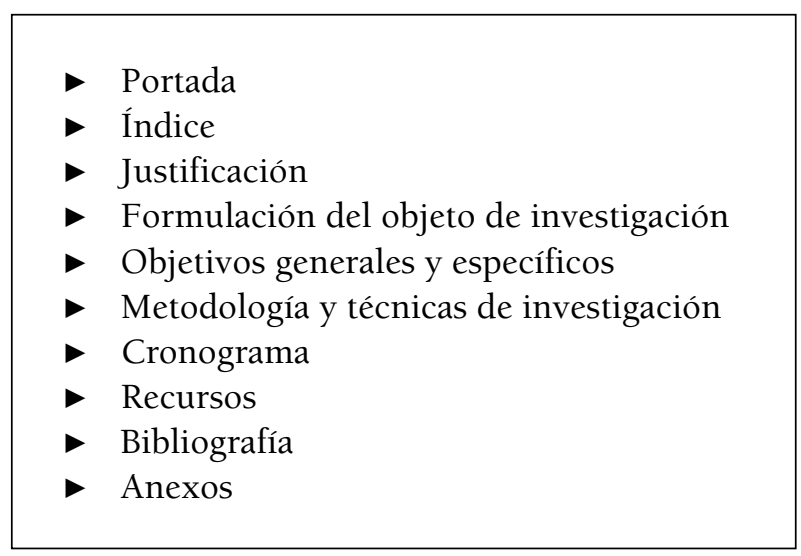

Fuente: Elaboración propia.

Los resultados derivados del análisis de cuestionarios se observó el interés por la investigación social. En ambos grupos casi el $90 \%$ la valoraron positivamente, considerando el aporte de este tipo de asignaturas en la titulación de Trabajo Social. En sus respuestas, justificaban la importancia de este conocimiento atendiendo las posibilidades de mejora en la elaboración de diagnósticos. Un destacado porcentaje (más de 50\%) incidió en la relevancia de la investigación previa de los fenómenos sociales y el interés que requería la observación y análisis de la realidad social. Así mismo otros alumnos manifestaban la importancia de la investigación atendiendo al prestigio que aportaba a la profesión desde el ámbito científico. No obstante, estos términos se evidenciaron con mayor proporción en los alumnos de último curso de Grado que en los de Nivelación, en los que incluso un pequeño número (2\%3) expresó que la investigación no

4. En este modelo básico de PIS se basó el taller colaborativo con los alumnos y sobre el mismo se evaluó su adquisición de competencias. 
era relevante para la intervención en Trabajo Social. Los alumnos de nivelación refirieron tener mayores carencias en investigación para la elaboración de diagnósticos efectivos que los alumnos de último curso de grado, los cuales sentían mayores dificultades para enfrentarse a la realidad de la intervención social.

Figura 3: Incidencia de la Investigación en Trabajo Social para la intervención con individuos, grupos y comunidades

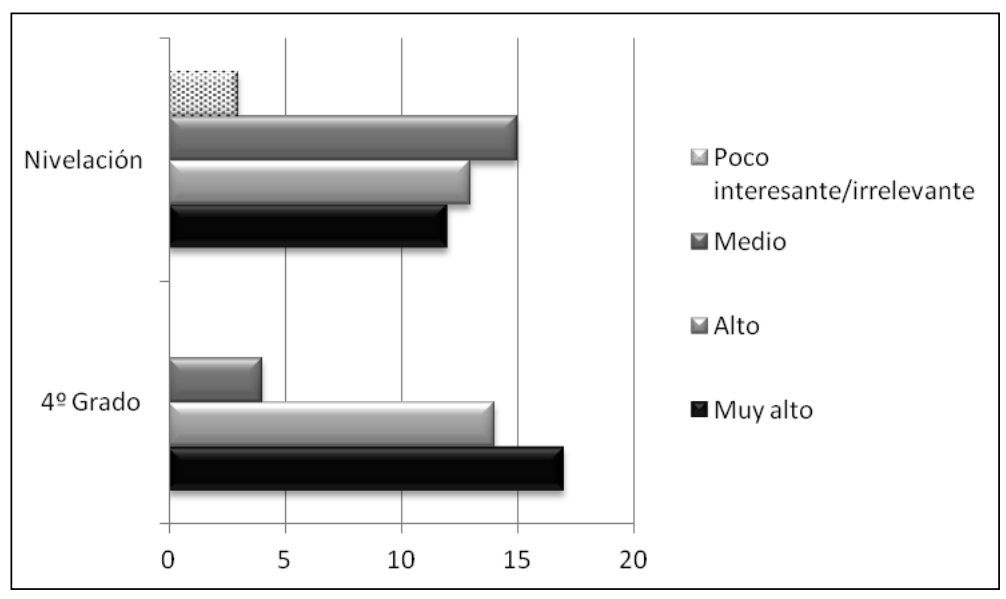

Fuente: Elaboración propia

Analizando otras cuestiones sobre las que ambos grupos coinciden (valoración positiva de la investigación para afrontar situaciones cotidianas de la profesión, relación del TFG con la investigación, perspectivas personalesprofesionales en referencia a la investigación...) e informados del porcentaje de créditos en investigación que tiene la titulación de grado de la Universidad de Valencia, más del 75\% de ambos grupos consideraron imprescindible incrementar la carga lectiva en esta materia. Las evidencias resultaron mucho mayores en los estudiantes de último curso de grado que en los de nivelación; ya que en estos últimos se apreciaron respuestas más heterogéneas, diversificando el incremento de la carga lectiva, orientada a la adquisición de competencias en investigación en la horquilla entre el 20\% y el 50\%. Expresaron que en este aumento dedicarían un importante espacio a los PIS y al trabajo diagnóstico. 
Figura 4: Interés por incrementar la carga lectiva en competencias de investigación

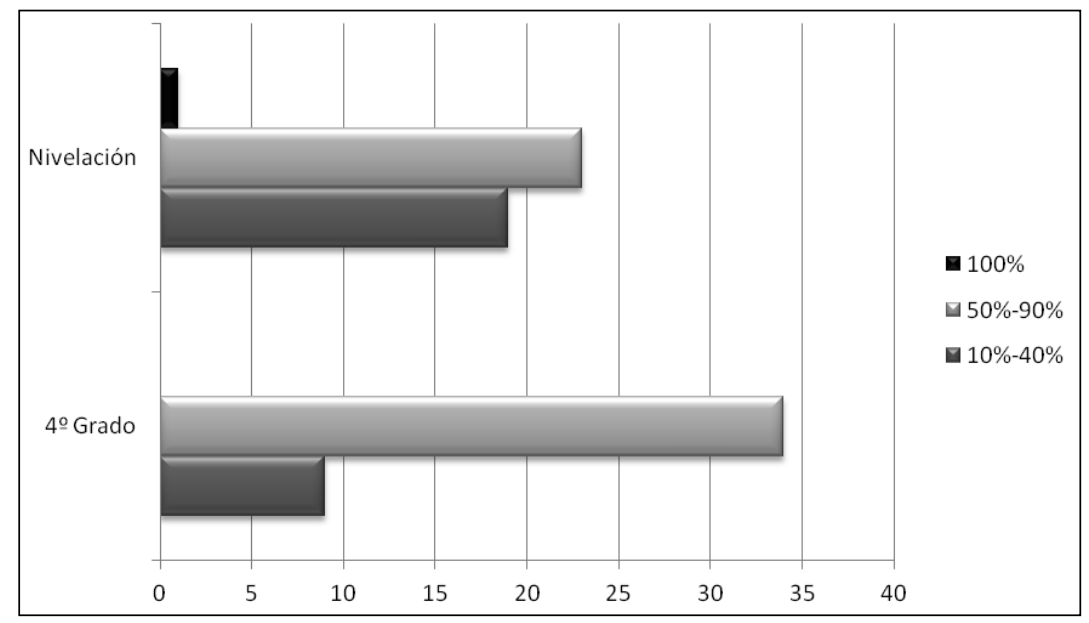

Fuente: Elaboración propia.

Los resultados derivados del análisis presentan la importancia que para ambos colectivos tiene las competencias investigadoras de los fenómenos sociales, pero hay que señalar que un $25 \%$ de los profesionales encuestados asumió la realización de diagnósticos sociales sin previa investigación, justificándola en primer lugar por la inmediatez que de su actividad cotidiana requieren determinadas respuestas, en segundo lugar por la dificultad del escenario profesional cotidiano que impide reflexionar sobre el trabajo que se realiza y por último, en el bagaje profesional que proporciona conocimientos previos, experiencias de desempeño y de la propia práctica; es decir, investigaciones anteriores, a veces, no sistematizadas.

Figura 5: La investigación en Trabajo Social como metodología para las buenas prácticas en el espacio profesional

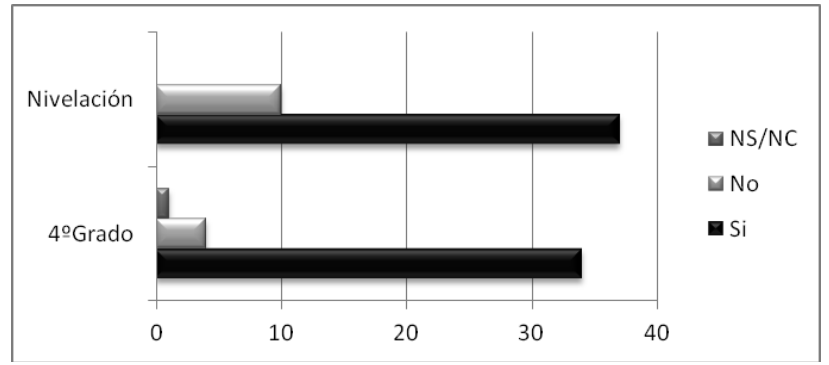

Fuente: Elaboración propia. 
En lo que se refiere a la valoración de competencias en investigación (Fig. 6) ambos grupos consideraron más importantes las vinculadas con la intervención (ítems I) y en menor medida las metodológicas (ítems M) y transmisión científica (ítems R). Mientras que en lo que se refiere a su percepción de competencias adquiridas los profesionales se sintieron más inseguros con las metodológicas (ítems M) y los estudiantes de Grado hicieron referencia a las propias de la de intervención. Es representativo como en ambos grupos despertó elevado interés la formación en metodologías científicas propias que pudieran permitir el desarrollo experimental del Trabajo Social. Respecto las competencias entre ambos, destacaron la similitud de pareceres en la redacción eficaz y el desarrollo de habilidades para analizar y sistematizar datos. Sin embargo las diferencias principales consistieron en la prioridad que unos y otros ofrecieron a la intervención sobre la investigación. Mientras que los estudiantes de último curso que carecían de experiencia profesional se posicionaron con mayor inquietud frente a la intervención y con mayor serenidad frente a la investigación, los estudiantes de nivelación ofrecieron resultados opuestos, atendiendo a la seguridad que confiere el espacio profesional basado en la experiencia y la inseguridad que representaba para ellos el escenario empírico. Asimismo los estudiantes de nivelación encontraron mayor interés que los de grado en poder relacionar la teoría del trabajo social con la intervención profesional pre diagnóstica. En relación a las competencias derivadas de la inmersión en bases de datos bibliográficas, los estudiantes de grado puntuaron interés superior y afrontaron tales ejercicios con mayor diligencia y práctica que los profesionales. 
Figura 6: Percepción de adquisición de competencias

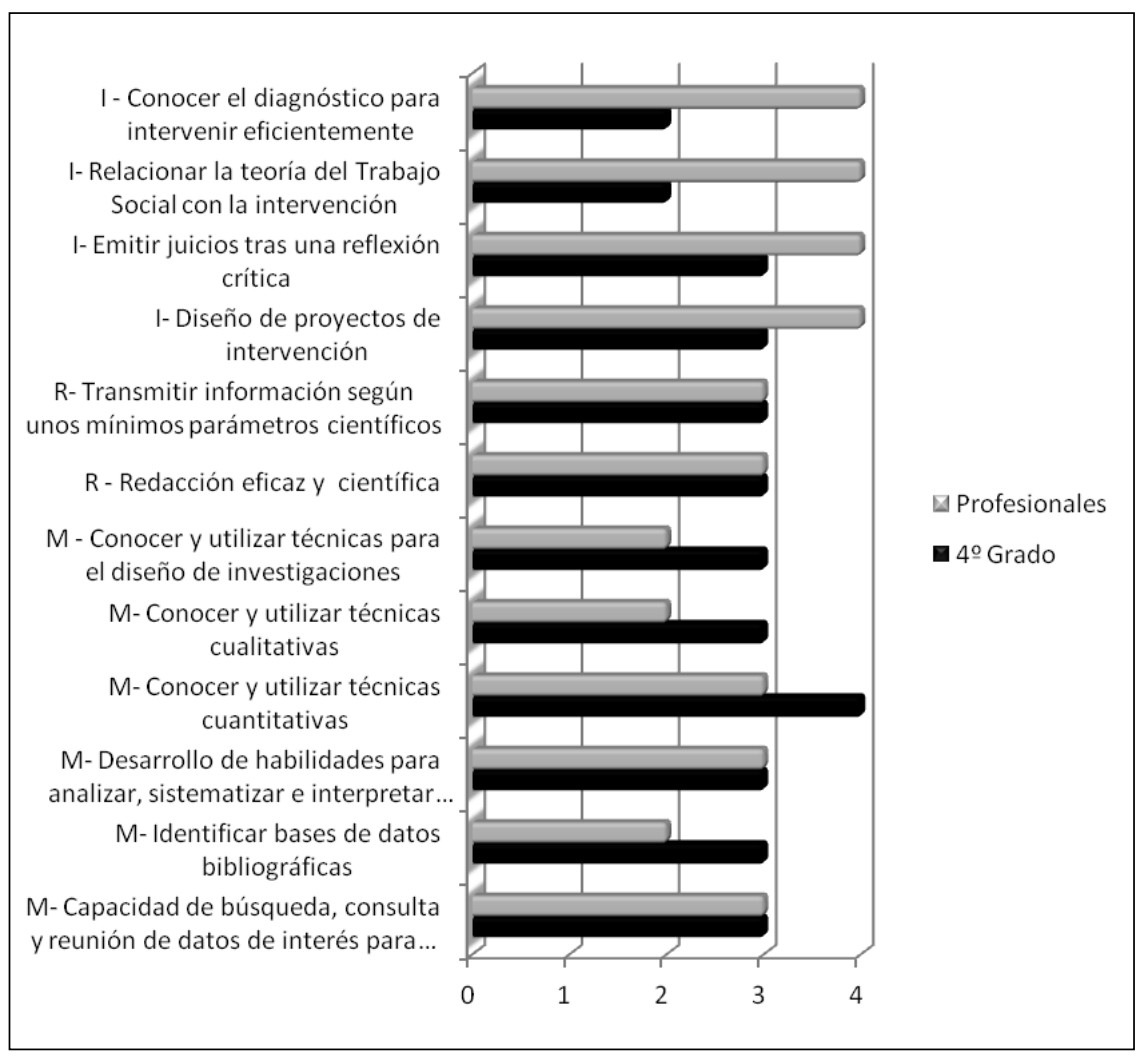

Fuente: Elaboración propia.

Finalmente, tanto alumnos como profesionales, en aproximadamente un $80 \%$ de los encuestados, enunciaron correctamente los proyectos de intervención, si bien en la formulación de los de investigación tan solo el $60 \%$ lo hizo de manera adecuada en el colectivo profesional y poco más del $40 \%{ }^{5}$ en el de estudiantes.

5. Este porcentaje ascenderá al 93\% tras la realización del taller de diseño de proyectos de investigación, indicativo que refleja la importancia de esta práctica dinámica y positiva para el aprendizaje y adquisición de las competencias señaladas.

Alternativas. Cuadernos de Trabajo Social, 23, 2016, pp. 71-90 - ISSN 1133-0473

DOI: 10.14198/ALTERN2016.23.04 
Figura 7: Competencias adquiridas en la formulación de proyectos de investigación (PIS) y de intervención

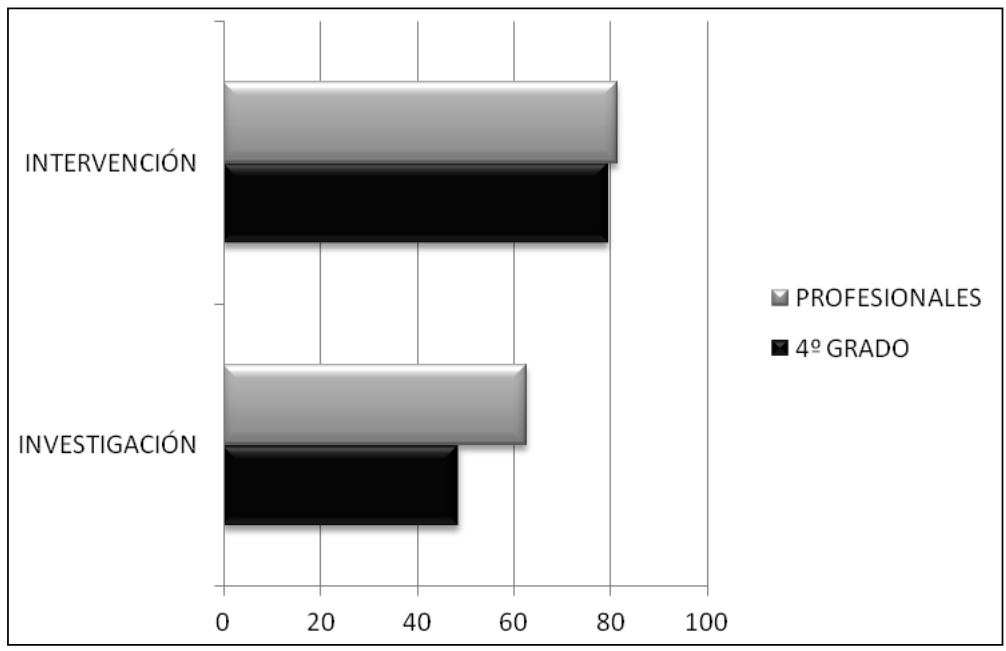

Fuente: Elaboración propia.

\section{Discusión}

El Trabajo Social necesita competencias altamente fiables para resolver los problemas de individuos, grupos y comunidades. El cambio social de los nuevos tiempos demanda respuestas inmediatas y convierte al Trabajo Social en una disciplina exigida por rápidas reacciones adaptadas a las necesidades sociales. Durante décadas se identificó la intervención como un tipo de práctica rutinaria con sentido esencialmente transformador (Erstad, 2002 y Bueno, 2013) y es aquí donde en estos momentos se asientan muchas de las estrategias del Trabajo Social, entre ellas, ofrecer respuestas urgentes ante las contingencias cotidianas. Contrario a lo que recomiendan los manuales de investigación social, que según Rubin y Babbie (2011) requiere de pausa y reflexión para el transcurrir de los hechos.

Los resultados evidencian puntos de encuentro y desencuentro entre los diferentes grupos de análisis. Ambos insistían en la prioridad de formarse para investigar incidiendo en la importancia de articular un título de Grado en Trabajo Social donde las materias relativas a investigación se coticen al alza, coherente con lo que se expresa en el Libro Blanco del Trabajo Social ya que las principales carencias en la formación se centran "en el análisis de la realidad (41,3\%), especialmente en lo referido a la aplicación de la metodología y 
técnicas de investigación y el diagnóstico de las situaciones sociales susceptibles de intervención" (2005: 224).

Es cierto que la investigación desde la esfera académica ha sido objeto de crítica desde las profesiones aplicadas, arengando estas mayor proximidad al objeto de estudio (Lozano, 2012). Con el ánimo de suplir estas distancias, coincidimos con Ibarra y Rodríguez (2010) en la necesidad de transformar tanto el modelo de enseñanza como el de evaluación universitaria, siendo prioritario el desarrollo de estrategias que favorezcan el contacto con la realidad social donde poder aplicar las competencias adquiridas; las mejoras en la formación han de introducirse atendiendo aspectos relacionados con el "conocimiento y el manejo de las técnicas y herramientas de investigación social (40,4\%), siendo éste un aspecto fundamental para poder llevar a cabo un adecuado análisis de la realidad" (Libro Blanco, 2005: 227). Sobre estas recomendaciones, los Grados universitarios de Trabajo Social denotan la importancia en la adquisición de competencias en investigación y en un alto porcentaje de las titulaciones consultadas, el PIS cumple la función de TFG, que converge con los datos derivados del estudio realizado por Carmona et al., (2012) focalizando la relación entre teoría y prácticas.

Regresando a nuestro objeto de análisis, ambos grupos destacan la necesidad de investigar para construir una práctica más efectiva desde el ejercicio profesional. Coincidiendo con las evidencias de Beltrán (2003), una mayoría de los encuestados alega alta ascendencia sobre los fenómenos sociales y el interés que requiere la observación de la realidad social para la intervención. Los datos reflejaron que una cuarta parte de los estudiantes del grupo de nivelación admitieron realizar diagnósticos en su trabajo cotidiano sin someter a investigación las evidencias y hallazgos encontrados.

La realización específica de diseño del PIS remarcaría el interés por la sistematización de las intervenciones y el establecimiento de un guión orientativo, permitiendo disponer de una interpretación más profunda. En la línea de los hallazgos de Botija y Navarro (2015) esto ayudaría a comprender y mejorar la práctica, serviría además para teorizar, generalizar y extraer enseñanzas para poder compartirlas en diferentes planos y momentos. Coincidiendo con Crisp (2015), la sistematización permite reflexionar sobre el desarrollo de la práctica profesional y mejorar las experiencias de aprendizaje curricular en el Grado en Trabajo Social.

La experiencia en contacto con la realidad social de uno de los grupos como el nulo contacto de otros o limitado a las prácticas preprofesionales, condicionaron el nivel de respuesta referida a la autopercepción de competencias; encontrándose los profesionales cómodos en la práctica pero limitados en la 
investigación frente a los alumnos de último curso que se situaron de manera inversa. Estos datos coinciden con las investigaciones de Pendler (2013) al detectar que los profesionales hallan su principal dificultad en las deficiencias en la metodología de investigación mientras que los alumnos adheridos al nuevo EEES se sienten con mayor seguridad en la adquisición de estas competencias. En consonancia, los profesionales de nivelación mostraron menos inseguridad en la intervención que los alumnos de último curso de grado.

\section{Conclusiones y propuestas}

En el nuevo Trabajo Social, la investigación constituye una actividad curricular básica de alumnos y docentes, que implica asociarse, socializar y compartir experiencias, siendo un reto la posibilidad de afianzar y proteger el espacio profesional. Además, se presenta otro desafío, donde el trabajo social no ha de reducirse solo a aquellos aspectos propiamente epistemológicos sino que también ha de prolongarse a otros que afectan todo proceso de investigación y converger con otras disciplinas, véase: procesos de acompañamiento, la gestión y orientación de las organizaciones, la educación social, los requerimientos de las nuevas estructuras sociales-familiares y de los escenarios de socialización educativa (escuela), los cuidados profesionales,... etc.

La investigación se reafirma como un componente más del proceso de intervención, por tanto, no separado de él. Las condiciones del proceso, el contexto y la estructura en la cual se desarrollan las prácticas profesionales, con la emergencia que exigen los procesos de crisis y la inmediatez de respuestas, obligan al Trabajo Social a planificar su modus operandi para de esta manera invertir en el resultado final. Ello implica analizar e investigar todas las situaciones para disponer de más puntos de vista para el abordaje de las problemáticas.

La competencia profesional para investigar y afrontar la realidad social es uno de los principales resultados que nos deja la presente investigación: es necesario ser más competentes en investigación, porque ello permitirá conocer mejor las reacciones de los individuos ante los fenómenos sociales. Los datos refieren que tanto los estudiantes de grado como los de nivelación se encuentran motivados para recibir formación en investigación y muestran interés en esta faceta para perfeccionar su actividad profesional, advirtiendo la importancia del proceso metodológico en la consecución de objetivos propuestos..

La intervención y en concreto el proyecto de intervención social es un elemento claramente definido e identificado frente al PIS, pese a ser éste parte necesaria del primero. Utilizar el PIS como TFG puede ser adecuado pero también ambicioso. Por ello es fundamental el aporte de un material claro 
y pertinente así como impartir una materia docente específica que acredite colaborativamente las carencias prácticas que presenta el alumnado. Por medio del diseño del PIS se puede evidenciar la adquisición de competencias y los procesos de enseñanza-aprendizaje a los que serían sometidos los estudiantes.

El hecho de investigar sobre el colectivo de estudiantes con experiencia en el ejercicio profesional del Trabajo Social ha corroborado que aquellos trabajadores sociales alejados de las metodologías de investigación, recurrían a ellas para conseguir mayor calidad y fiabilidad a su trabajo. Por otro lado, la investigación relativa a la práctica exige altas competencias en los nuevos graduados en Trabajo Social. Coincidimos con Erstad (2002), que integrados en la investigación desde dentro, se favorece el análisis de la realidad con objeto de generar teoría desde la práctica.

Las propuestas que nos deja el presente análisis se orientan hacia el desafío de explorar la relación conceptual entre experiencia práctica y experiencia teórica, factor que habrá de posibilitar la apertura a nuevos escenarios para la investigación y especialmente en las estrategias de investigación - acción - transformación. Conocer la realidad de estos espacios nos ayudará a incidir en las competencias necesarias y con ello, especializar más las herramientas y diseños de investigación propios del Trabajo Social. Por otro lado, queda demostrada la eficiencia en la adquisición de competencias para el diseño de PIS tras la realización de un taller práctico, configurado mediante técnicas de aprendizaje colaborativo.

Invertir en investigación afianza las expectativas de mejora de los profesionales del Trabajo Social. La integración en el curriculum transversal de estos aprendizajes permite afianzar un conjunto de complementos teórico-prácticos empírico-exploratorios. En definitiva, las competencias del profesional del Trabajo Social emergen desde una doble perspectiva, una primera de búsqueda con efectos diagnósticos y otra inspirada en la intervención y la transformación social en clave de desarrollo humano.

\section{Bibliografía}

Ahmed Mohamed, K. (2009). "El Trabajo Social ante el Espacio Europeo de Educación Superior: ¿Redefinición o continuidad?” Portularia, Vol. XIX, 1, 25-32.

Álvarez, P. (2015). Parejas y matrimonios mixtos: aspectos sociales y psicosociales de su configuración y desarrollo, Tesis Doctoral, Universidad de Huelva.

Álvarez, V. Azcárate, M.P., García, E., Gil, J., Ibarra, M.S, Rodríguez, G. Rodríguez, J. Serrado, A. y VALVERde, A. (2001). "Investigación sobre educación universitaria", Revista de Investigación Educativa, Vol. 19, 2, 615-631. 
ANECA (2005). Libro Blanco: Título de Grado en Trabajo Social. Madrid: Agencia Nacional de Evaluación de la Calidad y Acreditación, Madrid, ANECA-Agencia Nacional de Evaluación de la Calidad y Acreditación. http://www.aneca.es/ var/media/150376/libroblanco_trbjsocial_def.pdf (Consulta: 12 de febrero de 2015)

BARNER, J.R. y D. OKECH (2013) 'Teaching Globalization to Social Work Students: In and Out of the Classroom', Social Work Education Vol. 32, 8, 1061-74.

Bath, D., Smith, C.D., Stein, S. y Swann, R. (2004). "Beyond mapping and embedding graduate attributes: Bringing together quality assurance and action learning to create a validated and living curriculum", Higher Education Research and Development, Vol. 23, 3, 313-328.

BeltrÁn, M. (2003). La realidad social, Madrid, Tecnos.

BIGGS, J. y TANG, C. (2011). Teaching for quality learning at university, London, McGraw-Hill International.

BotiJa, Ma M. y NAVARro, J.J. (2015). "El proyecto de investigación social como instrumento integrador de la praxis para los futuros/as trabajadores/as sociales", Cuadernos de Trabajo Social, Vol. 28, 1, 47-58.

Bourdieu, P. (1999). La miseria del mundo, Madrid, Akal.

BRYdon, K. (2012). Promoting Diversity or Confirming Hegemony? In Search of New Insights for Social Work', International Social Work Vol. 55, 2, 155-67.

BuEno, A.M. (2013). "Producción de conocimiento sobre investigación en Trabajo Social", Trabajo Social, 15, 125-141

Callejo, J. y Viedma, A. (2006). Proyectos y estrategias de Investigación Social: La perspectiva de la intervención, Madrid, McGraw-Hill

Campanini, A. (2008). "Desarrollo y perspectivas de los estudios de Trabajo Social en Europa. Las Organizaciones Internacionales de Trabajo Social”, Humanismo y Trabajo Social, 7, 109-119

Carmona, M. Soria, V. y Nuri, A. (2012). "El Trabajo Final de Grado: una guía orientativa para los estudiantes", Actas VII Congreso Internacional de Docencia Universitaria e Innovación, Barcelona, 4- 6 de julio.

Chourides, P; LongbotTom, D. y Murphy, W. (2003). "Excellence in knowledge management: an empirical study to identify critical factors and performance measures", Measuring Business Excellence, Vol. 7, 2, 29 - 45

CRISP, B. R. (2015). "The challenges in developing cross-national social work curricula", International Social Work, Vol. 58, 1, 1-13

DíAz, A. (2014). "Construcción de programas de estudio en la perspectiva del enfoque de desarrollo de competencias", Perfiles Educativos, Vol. XXXVI, 143, 142-162.

ERSTAD, H. (2002). "La investigación en la práctica del Trabajo Social. La experiencia como fuente de conocimientos", Alternativas. Cuadernos de Trabajo Social, 10, 413-425. 
FERNÁNDEZ, M.A. (2015). La Competencia Intercultural en el marco de los Servicios Sociales Comunitarios, Tesis Doctoral, Universidad de Huelva.

García-SAn Pedro, M.J. (2009). "El Concepto de competencias y su adopción en el contexto universitario", Alternativas. Cuadernos de Trabajo Social, 16, 11-28.

IBARRA, M.S. y RODRÍGUEZ, G. (2010). "Aproximación al discurso dominante sobre la evaluación del aprendizaje en la universidad”, Revista de Educación, 351, 385-407.

Liedgren, P. (2015) "Transfer of Teaching Styles: Teaching Social Work in Iraqi Kurdistan as a Swede", International Social Work Vol. 58, 1, 175-185.

LOZANO, M. (2012). La Universidad como espacio y práctica de aprendizaje, Teknokultura, 9, 2, 121-130.

MARTín, M. (2013). La construcción de la identidad en los trabajadores sociales, Tesis Doctoral, Universidad Complutense de Madrid.

MARTínEZ, S. (2000). Herramientas para diseñar un proyecto de investigación social, Bucaramanga, Sic Editorial Ltda.

MCKEACHIE, W. (2002). Teaching tips: strategies, research and theory for college and university teachers, Boston: Houghton Mifflin.

Medina, A.M., Domínguez, M.C. y SánChez-Romero, C. (2013). "Evaluación de las competencias de los estudiantes: modelos y técnicas para la valoración", Revista de Investigación Educativa. Vol. 31, 1, 239-255.

Miranda, M. (2003). Pragmatismo, Interaccionismo simbólico y Trabajo Social. De cómo la caridad y la filantropía se hicieron científicas. Tesis Doctoral. Universitat Rovira i Virgili.

Molx, M. (2006). Teoría del Trabajo Social, Madrid, Síntesis.

Palacios, J.E., Cordero, N., Fernández, M.I. y Fernández, A.M. (2006). "Valores profesionales en los alumnos de Trabajo Social de la Universidad Pablo Olavide de Sevilla", Documentos de Trabajo Social: Revista de Trabajo y Acción Social, 38, 33-68.

PASTOR, E. (2008). La participación ciudadana en los Servicios Sociales municipales de la Región de Murcia, Murcia, Universidad de Murcia.

PASTOR, E. y SÁnCHEZ-Millán, M. (2014). "Analysis and Impact of the Economic Crisis and Regulatory Changes in the Needs and Benefits System Municipal Social Services: Analysis Case of Murcia-Spain", Revista de Cercetare si Interventie Sociala, Vol. 47, 7-31.

Pendler, M. (2013). Action learning in practice. London, Gower Publishing. PÉrez-Cosín, J.V. (2003). El Trabajo Social, sus imágenes y su público. La construcción de la identidad colectiva, Tesis doctoral, Universitat de València.

Richmond, M. E. (1917). Social diagnosis, New York, Rusell Sage Foundation. RODrígueZ-BERrio, A. (2000). "La singularidad de la investigación en Trabajo Social", en Dolors Colom y Miguel Miranda (coord.), Cambio social, relaciones 
humanas, nuevas tecnologías: enfoques para una formación de futuro, Zaragoza, Mira Editores, Vol. I, 139-144.

RubBin, A. y BABbIE, E.R. (2011), Research Methods for Social Work. Empowerment Series, Belmont (USA): Pre Press PMG.

Rubio, M.J. y VARAS, J. (2004). Análisis de la realidad en la intervención social, Madrid, CCS.

VÁzQUEZ, O. (2011), "Las competencias profesionales en los títulos de Grado en Trabajo Social”. Revista de Servicios Sociales y Política Social, 96, 21-36.

Williams, J. y Nelson, P. (2007) "SWIPE "Social Work International Practice

Education": European Collaboration to Develop a Module for Internationalising the Social Work Curriculum", European Journal of Social Work 10, 1,120-123. ZABAlZA, M.A. (2002). La enseñanza universitaria: el escenario y sus protagonistas, Madrid, Narcea. 